\title{
Editorial
}

\section{Mobile Assistive Technologies}

\author{
Simone Spagnol 1 , ${ }^{1}$ Ádam Csapó $(\mathbb{D})^{2}$ \\ Evdokimos I. Konstantinidis, ${ }^{3}$ and Kyriaki Kalimeri ${ }^{4}{ }^{4}$ \\ ${ }^{1}$ Department of Information Engineering, University of Padova, 35131 Padova, Italy \\ ${ }^{2}$ Department of Informatics, Széchenyi István University, Györ 9026, Hungary \\ ${ }^{3}$ Lab of Medical Physics, Medical School, Aristotle University of Thessaloniki, 54124 Thessaloniki, Greece \\ ${ }^{4}$ Data Science Laboratory, ISI Foundation, 10126 Torino, Italy \\ Correspondence should be addressed to Simone Spagnol; spagnols@hi.is
}

Received 26 March 2018; Accepted 26 March 2018; Published 28 May 2018

Copyright (c) 2018 Simone Spagnol et al. This is an open access article distributed under the Creative Commons Attribution License, which permits unrestricted use, distribution, and reproduction in any medium, provided the original work is properly cited.

Over the past few years, assistive technologies have achieved important milestones in enhancing the quality of everyday life, in terms of both autonomy and well-being, of individuals in need of assistance and care. The convergence process behind ICT in the past decades, mainly from the point of view of technology, but also in terms of management and regulation has led to the widespread availability of digital tools and rich data sources as building blocks for effective solutions towards assistive technologies. Today the promise of technologies that allow individuals to benefit from portable and discrete aids delivered through mobile devices seems more relevant than ever before. Ranging from assistive home automation (domotics) solutions to applications that repurpose smartphones into electronic assistive devices, breakthrough technologies are allowing users with physical and/or mental disabilities to accomplish everyday tasks, as well as to learn and develop social skills more easily.

The goal of this special issue was to create a multidisciplinary forum of discussion on recent advances within the field of mobile technology to support individuals of all ages in need of assistance and care. The issue is comprised of five papers, each focusing on some aspect of rehabilitation, or on ways of mitigating the effects of disabilities through a unique combination of a variety of sensory and display technologies. The technologies of BCI, virtual reality, and auditory displays are especially well represented in the special issue.

The paper by A. Athanasiou et al. (entitled "Wireless Brain-Robot Interface: User Perception and Performance Assessment of Spinal Cord Injury Patients") presents a pair of 8-DoF anthropomorphic robotic arms that can be controlled through kinesthetic motor imagery using the commercially available Emotiv EPOC BCI device. Through an extensive set of user experiments, the authors provide a detailed assessment of how parameters such as age, independence, psychometric evaluation results, and neurological condition (i.e., among both spinal cord injury patients and healthy individuals) influences users' ability to control the system and their perception of the system. Based on the results, the authors conclude that BCI systems enabling users to effectively control multiple degrees of freedom in real-time may well be within short-term reach.

The paper by R. B. Lupu et al. (entitled "BCI and FES Based Therapy for Stroke Rehabilitation Using VR Facilities") also centers around BCI technology but combines it with virtual reality with a view towards supporting the rehabilitation of stroke patients. The paper introduces a system called TRAVEE, which makes combined use of an array of stimulation devices (e.g., functional electrical stimulation) to help the execution of the rehabilitation tasks, as well as monitoring devices (via BCI and electrooculography) and visual feedback (through virtual therapist) to provide users with real-time guidance. Based on a set of evaluations, the authors confirm that this closed-loop approach of connecting action, stimulation, and real-time feedback can go a long way in supporting the rehabilitation process, while at the same time alleviating the need for the continuous availability of trained therapists.

The paper by J. Gomez et al. (entitled "Using Smartphones to Assist People with Down Syndrome in Their Labour Training and Integration: A Case Study") presents a smartphone application called AssisT-Task, which was developed by the authors to help individuals with cognitive 
impairments to establish effective learning methods. The application is structured such that caregivers can define tasks that consist of sequences of subtasks and describe each subtask using text and visual figures which can then be retrieved through context-specific $\mathrm{QR}$ codes. A detailed analysis provided in the paper highlights the kinds of tasks and types of disabilities with respect to which the application provides the most benefits, and the authors conclude that, based on the evidence, the application may be most suitable to people with Down syndrome who are being trained to get a job.

The paper by by C. Saitis et al. (entitled "Cognitive Load Assessment from EEG and Peripheral Biosignals for the Design of Visually Impaired Mobility Aids") aims to develop an approach that is capable of real-time automated cognitive load analysis for visually impaired people. To that end, the paper investigates the ways in which different indoor and outdoor scenarios can be linked to certain characteristics of EEG sensor data (in particular, a slightly modified version of the ERD/ERS index, which is a well-established measure of cognitive load) in test subjects with different degrees of visual impairment. At the same time, a random forest based model is proposed that uses the measurements of electrodermal activity and blood volume pulse to predict discrete categories based on the ERD/ERS indices. The success of the model suggests that it may be possible to accurately gauge cognitive load using widely available, unencumbered sensors, while the scenario-dependent investigation in the first part of the paper highlights the fact that environmental and subjectspecific factors may serve as useful a priori information in the development of robust solutions for electronic travel aids (ETAs).

Finally, the paper by S. Spagnol et al. (entitled "Current Use and Future Perspectives of Spatial Audio Technologies in Electronic Travel Aids") focuses on a wide range of aspects relevant to the implementation of spatialized audio feedback in ETAs for the visually impaired. The paper provides a detailed overview on the acoustic and environmental parameters which influence the spatial perception of auditory stimuli, as well as the software and hardware aspects of spatial sound production, and the ways in which those approaches are used in currently available ETAs. In terms of software, special focus is given to the various ways in which headrelated transfer functions (HRTFs) can be described-that is, through generic models, personalized models, structurally motivated models, and analytical or numerical means. In terms of hardware, headsets that present sound without occluding the ears (allowing visually impaired users who rely on input from the physical environment to perceive it at the same time), such as bone conduction headsets and active transparent headsets, are highlighted as being most relevant to ETAs.

\section{Acknowledgments}

As guest editors, we would like to thank all the authors and reviewers whose contributions have made this special issue possible. It is our hope that the selection of works contained in the issue will serve as inspiration for further research and development in the area of mobile assistive technologies.

Simone Spagnol

Ádam Csapó

Evdokimos I. Konstantinidis

Kyriaki Kalimeri 


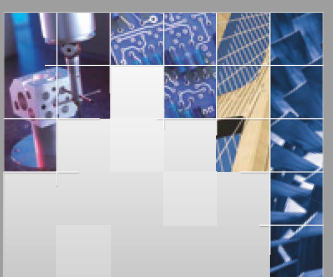

\section{Enfincering}
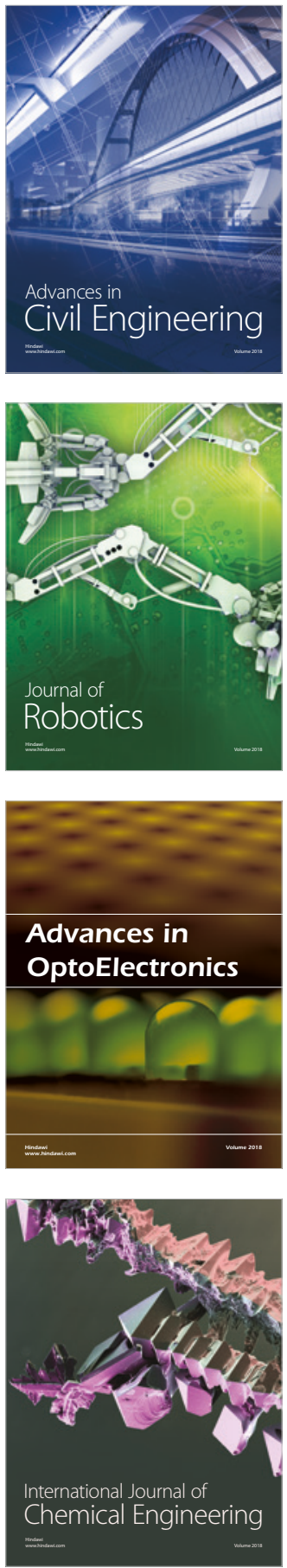

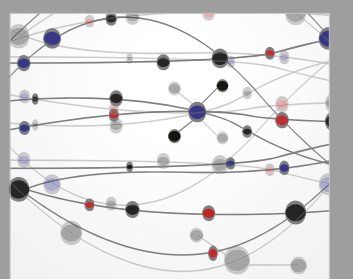

\section{Rotating \\ Machinery}

The Scientific World Journal

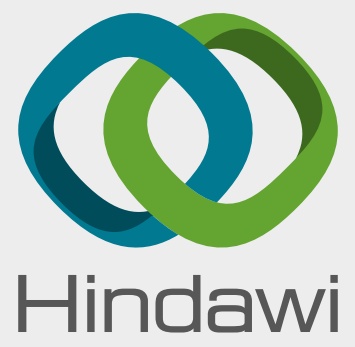

Submit your manuscripts at

www.hindawi.com
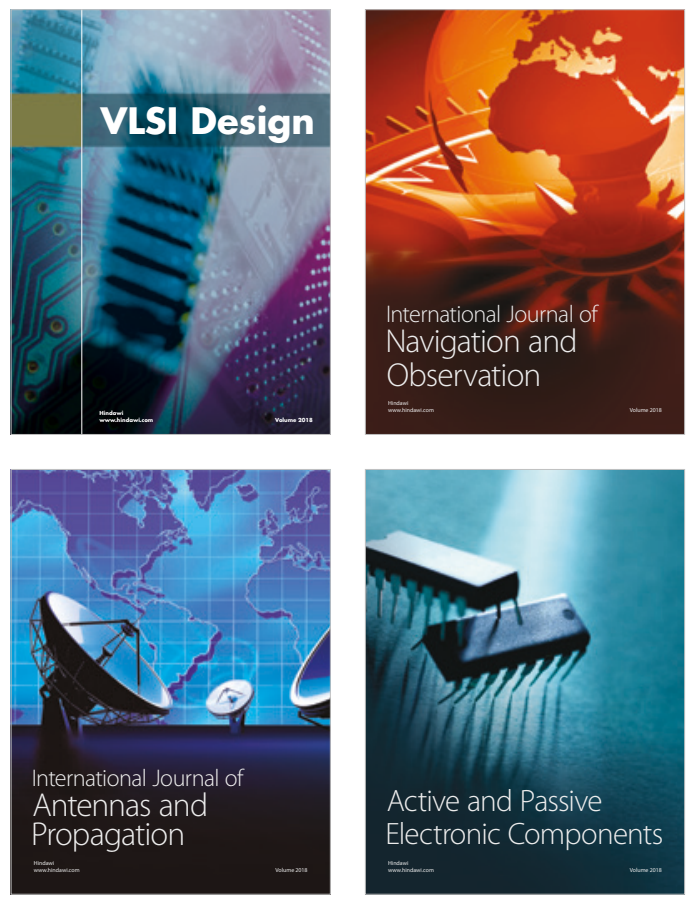
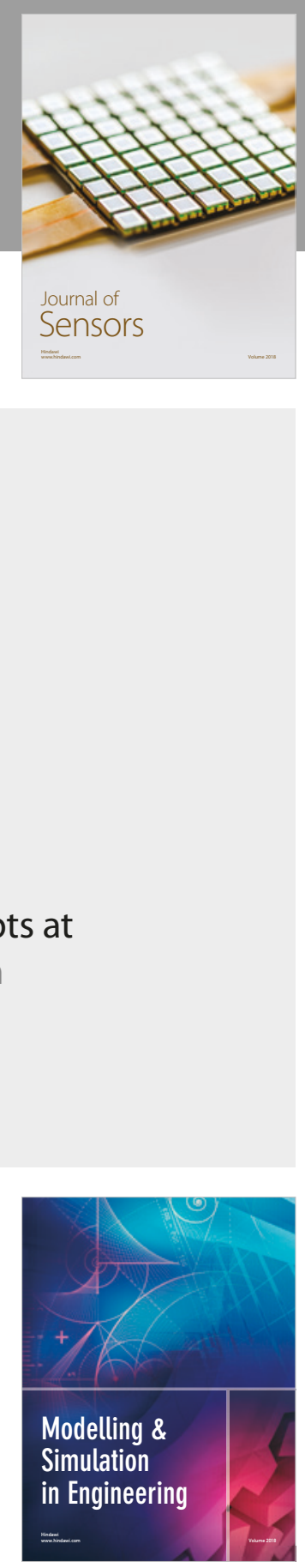

\section{Advances \\ Multimedia}
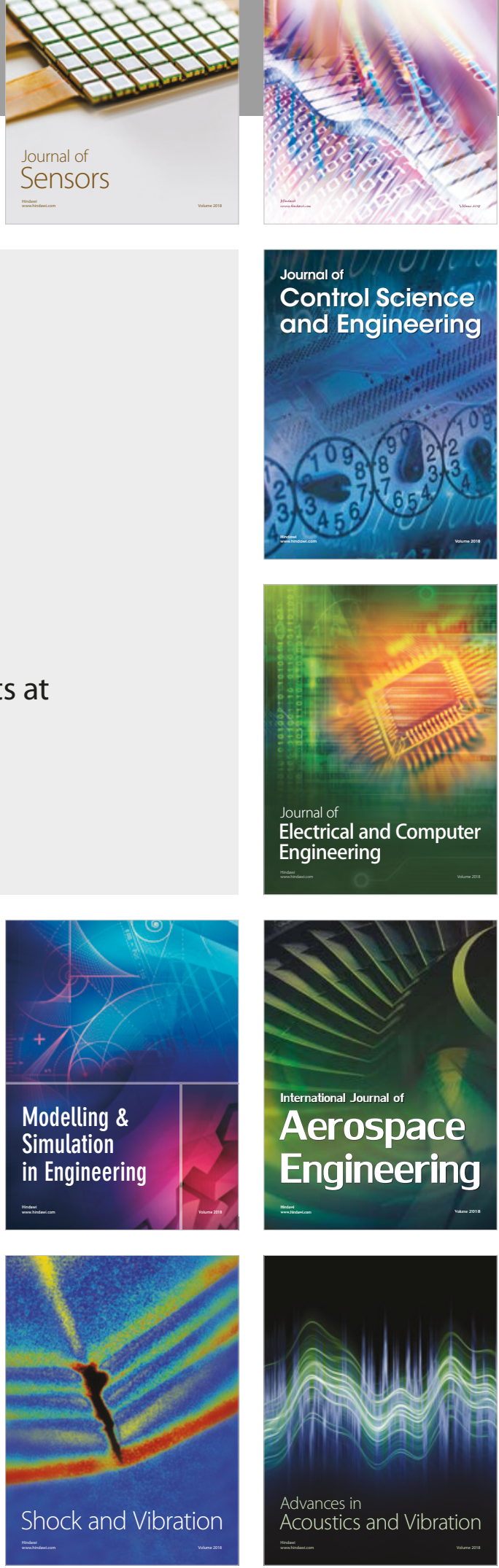